\title{
Metastatic Prostatic Carcinoma to the Testis: A Rare Case Report
}

\author{
Mohd Talha ${ }^{1}$, Sumbul Warsi ${ }^{2}$, Shafaque Zabin ${ }^{2}$, Kafil Akhtar ${ }^{3 *}$
}

\begin{abstract}
${ }^{1}$ M.D., Senior Resident, Department of Pathology, Jawaharlal Nehru Medical College, Aligarh Muslim University, Aligarh, Uttar Pradesh, India
${ }^{2}$ M.B.B.S., Junior Resident, Department of Pathology, Jawaharlal Nehru Medical College, Aligarh Muslim University, Aligarh, Uttar Pradesh, India

${ }^{3}$ M.D., D.M.R.T., Professor, Department of Pathology, Jawaharlal Nehru Medical College, Aligarh Muslim University, Aligarh, Uttar Pradesh, India
\end{abstract}

DOI: $\underline{10.36347 / \text { simcr.2020.v08i03.002 }}$

| Received: 19.02.2020 | Accepted: 26.02.2020 | Published: 07.03.2020

*Corresponding author: Dr. Kafil Akhtar

Abstract

Case Report

Prostatic carcinoma is one of the most common malignancy in aged males. The incidence of metastatic prostatic adenocarcinoma is well known with most common site being the bone followed by lung and liver. Metastasis to testis is rare and very few cases have been reported till date. We present a case report of 80 year old male who presented with swelling in the left testis since last 4 months with a history of radical prostatectomy performed 3 year back for resection of a malignant growth in the prostate. Left inguinal orchiectomy was done. Histopathological examination showed foci of atypical cells in acinar arrangement infiltrating into the tunica adventitia of the testis, suggestive of metastatic prostatic adenocarcinoma to the testis. Positive immunoexpression of AMACR and negative p63 expression further potentiated our diagnosis. This case report stresses the need for aggressive post-operative follow up in prostatic carcinoma patient and to consider the need of prophylactic orchiectomy in old patients.

Keywords: Adenocarcinoma, Metastasis, Orchiectomy, Prostate, Testis

Copyright @ 2020: This is an open-access article distributed under the terms of the Creative Commons Attribution license which permits unrestricted use, distribution, and reproduction in any medium for non-commercial use (NonCommercial, or CC-BY-NC) provided the original author and source are credited.

\section{INTRODUCTION}

Prostate carcinoma is the most commonly diagnosed carcinoma in the elderly male population of more than 50 years of age [1]. It is frequently an adenocarcinoma of acinar types with involvement of peripheral zone (posterior and lateral lobes) in most of the cases. In the early stage of the disease there may be no sign and symptoms, which develops in later stages of the disease due to compressive effects on the urethra with features of difficulty in urination, dribbling of urine, erectile dysfunction followed by bone pain in the terminal stages.

Most important risk factor is age and risk of prostatic malignancy increases with advancing age. It is diagnosed by a combination of digital rectal examination and serum prostate specific antigen (PSA) levels [2]. Extra prostatic invasion in seminal vesicle, bladder base and urethra is well known [3]. In later stage, frequent site of metastasis is pelvic group of lymph nodes followed by bone and the lung.

Early Radical prostatectomy is the primary treatment of choice owing to metastatic nature of tumour. It rarely metastasizes into the testis which is reported in about $0.5 \%$ cases only [4]. Semans' in 1938 published first case of prostate carcinoma with metastases into a testis [5]. Haupt et al., in 1984 reported 2 cases of occult malignancy with first manifestation as testicular mass [6]. They also reported that distinction of primary from metastatic testicular tumour in absence of previous diagnosis of primary poses a diagnostic challenge [6]. Metastatic deposit to the testis are more common from primary in the lung followed by the prostate and the gastrointestinal tract [7]. They are often detected incidentally after orchiectomy for advanced disease elsewhere [8]. We present this case report of a secondary testicular mass from primary prostate cancer due to its rarity and a sincere suggestion that a testicular mass should be investigated with a prompt suspicion so as to not miss a metastatic foci of a primary lesion.

\section{CASe Summary}

An 80 year old male presented to the surgery out-patient department with history of swelling in the left testis, bleeding per rectum and urinary incontinence since the last 4 months. The swelling was $2 \times 3 \mathrm{cms}$ in size, painless, firm to hard in consistency and associated with heaviness in the scrotum and not associated with any inguinal lymphadenopathy. His treatment history revealed radical prostatectomy performed 3 years back for prostatic carcinoma. Previous histopathology report showed the diagnosis of primary prostatic 
adenocarcinoma with gleason score of 8 without any evidence of metastasis. He was administered 50Gy of Co-60 radiotherapy and cisplatin 50mg x 6cycles of chemotherapy and subsequently advised routine assessment of serum PSA levels and imaging to rule out metastasis in the bone, liver and lung at 6 monthly interval.

General examination was normal except for mild pallor. Systemic examination was unremarkable. Abdominal examination revealed vague generalised pain in the lower abdomen with history of 1-2 episodes of vomiting. Examination of the left testis showed a mild tender $2.5 \times 2.5 \mathrm{cms}$ firm to hard swelling in the lower pole of the testis.

Routine examination like haemogram, general blood picture, urine examination, liver function test, renal function test, lipid profile, iron profile and electrocardiogram were within normal range except for presence of microcytic hypochromic anaemia owing to iron deficiency and recurrent rectal bleeding. Imaging studies viz., $\mathrm{x}$ rays of chest and abdomen showed normal studies. MRI and PET scan of abdomen were normal. CT scan of lower abdomen revealed a swelling in the left testis with involvement of the epididymis and the rete testis. Right testis was normal. Serum PSA, CEA, AFP and beta HCG were within normal limits.

A provisional diagnosis of testicular carcinoma was made and left radical orchiectomy was performed. Histopathological examination showed foci of atypical cells in acinar arrangement infiltrating into the tunica adventitia of the testis (Figure 1 and 2) with variable sized thick walled seminiferous tubules having few spermatocytic precursors and spermatids (Figure-3). Immunoexpression of AMACR was positive (Figure-4) while CK903 and p63 was negative (Figure-5). A final diagnosis of metastatic prostatic carcinoma to the testis was made. The patient was thereafter administered 6 cycles of anthracycline based chemotherapy and antiandrogen therapy for 6 months. Owing to old age andrisk of recurrent metastasis in right testis, patient underwent prophylactic right orchiectomy in the same operative settings. Follow up of the patient after 6 months revealed no metastatic disease and our patient is doing well.

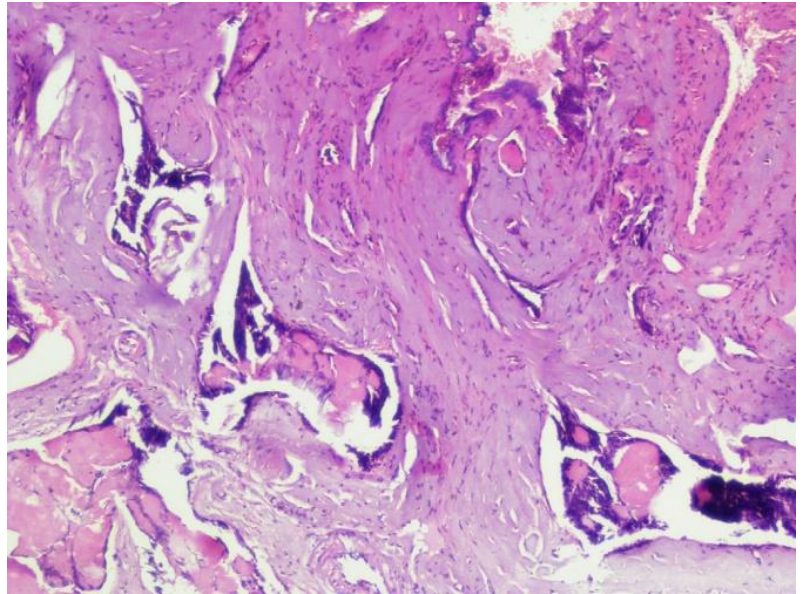

Fig-1: Histopathological examination of the testicular specimen showed foci of atypical cells in acinar arrangement infiltrating into the tunica adventitia of the testis (Haematoxylin and Eosin $x$ 10X)

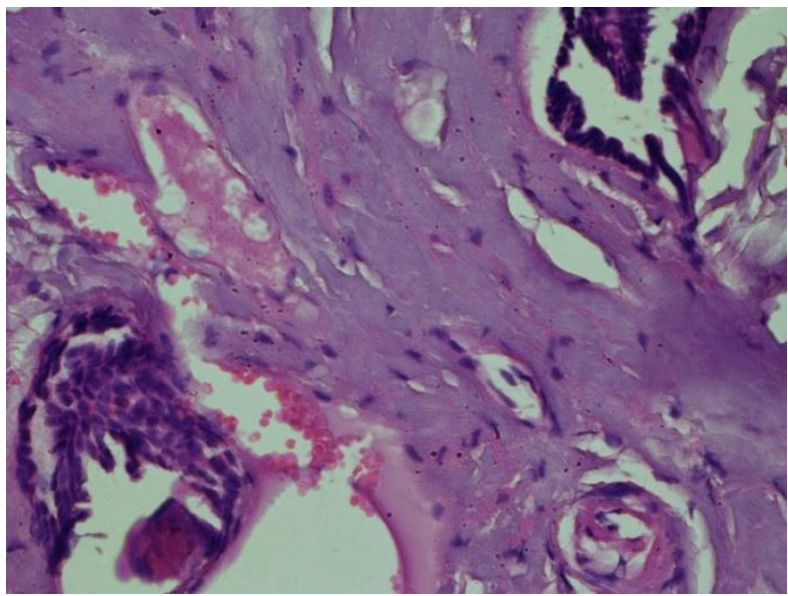

Fig-2: High power of Figure 1

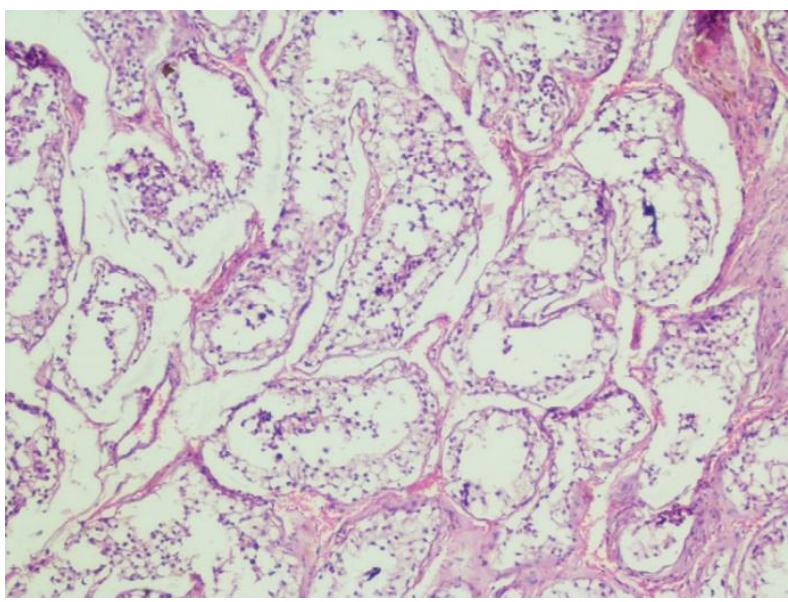

Fig-3: Tissue section shows variable sized thick walled seminiferous tubules having few spermatocytic precursors and spermatids (Haematoxylin and Eosin $x$ 40X) 


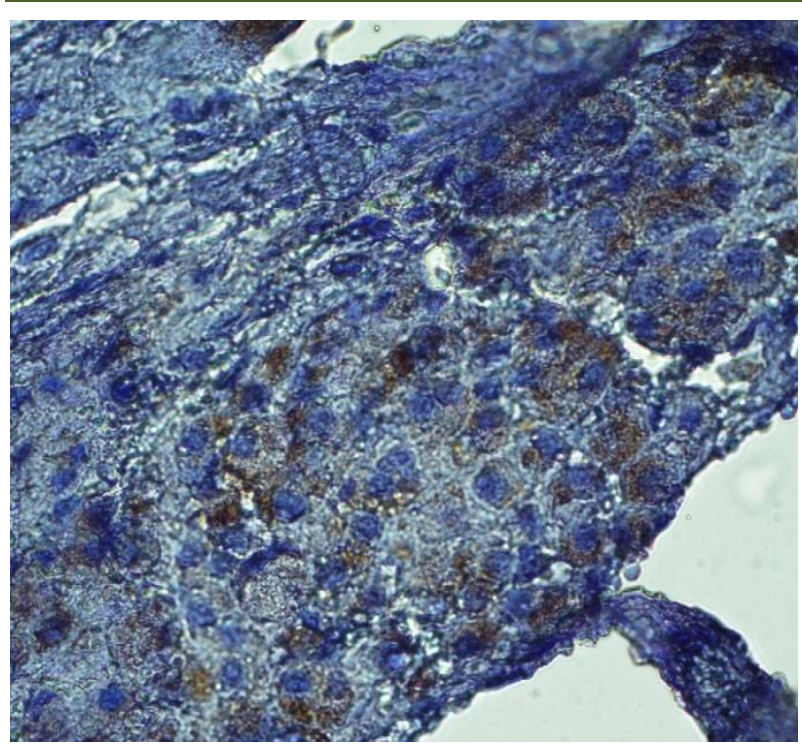

Fig-4: Immunohistochemical expression of AMACR showed cytoplasmic positivity (IHC AMACR x 40X)

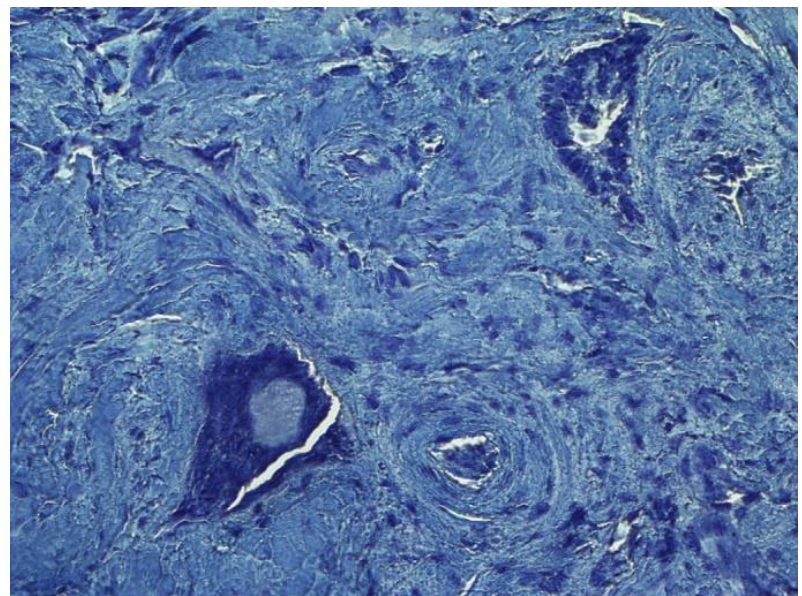

Fig-5: Immunohistochemical expression of p63 was negative (IHC p63 x 40X

\section{DISCUSSION}

Prostate is an integral part of male reproductive tract and contributes to the maximum volume of ejaculate. It weighs 7-16 grams in normal adults. It may undergo physiological hypertrophy with increasing age due to prolong exposure with androgens known as benign nodularhyperplasiawhich involve transitional zone while carcinomas are known to involve the peripheral zone. Nodular hyperplasia causes symptoms due to compressive effects on urethra while carcinoma prostate cause symptoms in late stages and are usually detected incidentally [9].

Various studies suggest that prolonged androgens exposure (old age), heredity (common in first degree relatives), ethnicity (more common in AfroAmericans), environmental factors and acquired somatic mutations (TMPRSS2-ETS gene fusions) have important roles in the pathogenesis and progression of prostate cancer [10].
Prostate cancers are mostly adenocarcinomas with well-defined glands. The glands are lined by a single uniform layer of cuboidal or low columnar epithelium with absence of basal cell layer seen in benign glands. Occasionally glands are crowded and may lack branching and papillary infolding. The cytoplasm is clear to amphophilic in appearance. Nuclei are enlarged and often contain one or more nucleoli. Pleomorphism is not marked and mitotic figures are uncommon [11].

The best way to diagnose prostatic cancer is to perform digital rectal examination (DRE) along with serial monthly serum PSA assessment in suspected individualswith high risk of malignancy [7]. Most prostate cancers are small, non-palpable, asymptomatic lesions discovered on needle biopsy performed to investigate an elevated serum prostate-specific antigen (PSA) level. Once prostate carcinoma is diagnosed and treated by radical prostatectomy, serum PSA is of great importance in predicting progression and prognosis of disease [7]. Metastasis is most commonly in bone followed by lungs and liver [2].

Secondary testicular tumours are mostly lymphomas and leukaemias. They usually are more common in old age group (50-60 years) and are mostly unilateral and rarely bilateral $[11,12]$. Metastasis to the testis may arise most commonly from the prostate followed by gastrointestinal tract, lung, kidney and rarely melanoma [7].

\section{Conclusions}

Prostatic adenocarcinoma is a dreadful malignancy in the aged males. Routine follow up after radical prostatectomy should be performed with monthly serum PSA and radiographic investigations to rule out recurrence/metastasis to other organs. This case emphasizes the fact that testicular mass should be approached with clinical suspicion of metastasis and asserts the importance of early prophylactic orchiectomy in older males with subsequent metastasis to the contralateral testis.

\section{REFERENCES}

1. Arnold M. Recent trends in incidence of five common cancersin 26 European countries since 1988: Analysis of the EuropeanCancer Observatory. Eur J Cancer, 2015; 11(51):11641166.

2. Ilic D, Djulbegovic M, Jung JH, Hwang EC, Zhou Q, Cleves A, Agoritsas T, Dahm P. Prostate cancer screening with prostate-specific antigen (PSA) test: a systematic review and meta-analysis. bmj. 2018 Sep 5;362:k3519.

3. Cheng L, Darson MF, Bergstralh EJ, Slezak J, Myers RP, Bostwick DG. Correlation of margin status and extraprostatic extension with progression of prostate carcinoma. Cancer: 
Interdisciplinary International Journal of the American Cancer Society. 1999 Nov 1;86(9):1775-82.

4. Bubendorf L, Schopfer A, Wagner U. Metastatic patterns of prostate cancer: an autopsy study of 1589 patients. Hum Pathol, 2000; 31: 578-583.

5. Semans JH. Carcinoma of the prostate with metastasis in the testis. J Urol, 1938; 40(4):524529.

6. Haupt HM, Mann RB, Trump DL, Abeloff MD. Metastatic carcinoma involving the testis. Clinical and pathologic distinction from primary testicular neoplasms. Cancer, 1984; 54(4):709-714.

7. Dutt N, Bates AW, Baithum SI. Secondary neoplasms of the male genital tract with different patterns of involvement in adults and children. Histopathol, 2000; 37:323-331.

8. Tu SM, Reyes A, Maa A, Bhowmick D, Pisters LL, Pettaway CA et al: Prostate carcinoma with testicular or penile metastases. Clinical, pathologic, and immunohistochemical features. Cancer, 2002; 94:2610-2617.

9. Foo KT. Pathophysiology of clinical benign prostatic hyperplasia. Asian J Urol, 2017;4(3):152157.

10. Epstein JI, Egevad L, Amin MB, Delahunt B, Srigley JR, Humphrey PA. The 2014 International Society of Urological Pathology (ISUP) consensus conference on Gleason grading of prostatic carcinoma. The American journal of surgical pathology. 2016 Feb 1;40(2):244-52.

11. Tiltman J. Metastatic tumours in the testis. Histopathol, 1979; 3: 31-37.

12. Manikandan R, Nathaniel C, Reeve N, Brough RJ. Bilateral testicular metastases from prostatic carcinoma. Int J Urol, 2006; 13: 476-477. 